\title{
Aufbau, Alter und regionale Einordnung der Endmoränen im Gebiet des Falken-Berges bei Fallingbostel (Niedersachsen)
}

\author{
Hans Dietrich Lang *)
}

Structural geology, end moraines, meltwater, discharge, glacial sedimentation, section, Saale Glaciation, Drenthe Stadial.

Northwestern German Plain, Falken-Berg Area, Lower Saxony, TK 25: Nr. 3024, 3214, 3125, 3224

$\mathrm{Kurzf}$ assung: In dem Hügelgebiet um den Falken-Berg zwischen Fallingbostel und Bergen $\mathrm{Kr}$. Celle zeichnen sich 3 Endmoränenbögen $\mathrm{ab}$, der Örbker, der Becklinger und der wenig deutlich ausgeprägte Kl. Bockeler Endmoränenbogen. Örbker und Kl. Bockeler Endmoränenbogen verdanken ihre Entstehung Gletscherloben, die von NW heranrückten; der Becklinger Endmoränenbogen geht auf einen von NE heranrückenden Lobus zurück.

Keine dieser Endmoränen ist vom nordischen Inlandeis überfahren worden. Ein nahe den Endmoränenbögen wurzelnder Fächer aus Schmelzwasser-Ablagerungen läßt sich bis nahe an die Aller-Niederung heran verfolgen. Er überlagert die drenthestadiale Hauptmoräne, ist also jünger als diese.

Die Endmoränen zwischen Fallingbostel und Bergen sind in einer späten Phase des DrentheStadiums der Saale-Eiszeit entstanden.

\section{[The Structure, Age and Regional Distribution of the End Moraines in the Falken Berg Area near Fallingbostel (Lower Saxony)]}

$\mathrm{Abstract}$ : In the hilly area around the Falken Berg, between Fallingbostel and Bergen, northwest of Celle, three end moraines are visible as arcuate ridges, the Orbbe and Becklingen end moraines, and the poorly developed Klein Bockel end moraine. The Orbke and the Klein Bockel end moraine were shaped by glacier lobes which advanced from the NW; the Becklingen end moraine is attributed to a glacier lobe from the NE.

None of these end moraines was overrun by the Scandinavian ice sheet.

A fan of melt-water deposits originates near the end moraines and can be traced nearly down to the edge of the Aller lowland. The fan deposits overlie and therefore postdate the main Drenthe ground moraine.

The end moraines between Fallingbostel and Bergen were formed in a late phase of the Drenthe Stadial of the Saale glaciation.

\section{Einführung}

Aus der relativ flachen und wenig gegliederten Altmoränenlandschaft der südlichen Lüneburger Heide mit Geländehöhen um 60-80 m üb. NN hebt sich das Areal um den Falken-Berg nahezu blockartig heraus. Dieses Hochgebiet hat die Form eines nach $\mathrm{N}$ zugespitzten Keiles, dessen N-S-Ausdehnung etwa $11 \mathrm{~km}$ und dessen Breite (von W nach E) an seinem S-Ende etwa $7,5 \mathrm{~km}$ beträgt. Der plateauartige nördliche Teil zeigt Höhen um $100 \mathrm{~m}$ üb. NN und wenig darüber, die höchsten Höhen liegen im S mit $150 \mathrm{~m}$ üb. NN. Dieses Hochgebiet fällt für Flachlandverhältnisse steil nach allen Seiten hin ab.

*) Anschrift des Autors: Dr. H. D. L a n g, Nieders. Landesamt f. Bodenforschung, Stilleweg 2, Postfach 5101 53, 3000 Hannover 51. 
Die erste geologische Darstellung dieses Gebietes findet sich bei STOLLER (1918) auf einer geologischen Übersichtskarte zu seinem Geologischen Führer durch die Lüneburger Heide, bereits mit dem Hinweis, daß es sich hier um eine Endmoräne der „vorletzten Vereisung“ handele. Der Name „Falkenberg-Endmoräne“ tritt erstmals bei WoLDSTEDT (1938) auf.

Die detaillierte geologische Erforschung dieses Gebietes hat lange hinter der anderer Gebiete hergehinkt, da es als militärisches Übungsgelände nur kurzfristig und in Ausnahmefällen betretbar ist.

Die hier vorgelegten Ergebnisse beruhen auf einer Kartierung der Blätter 3224 Westenholz und 3225 Offen der Geologischen Karte 1:25000, auf einer Auswertung alter Übersichts-Kartierungen auf den Blättern 3025 Soltau (NIEDERMAYER 1949), 3124 Fallingbostel (Niedermayer 1950a), 3125 Bergen (Niedermayer 1950b) eigenen Untersuchungen und Aufschlußbefahrungen im Falkenberg-Gebiet auf den Blättern 3025 Soltau, 3124 Fallingbostel und 3125 Bergen und der Auswertung von Luftbildern und von Archivunterlagen des Nieders. Landesamtes für Bodenforschung in Hannover.

Die Kommendantur des NATO-Schießplatzes Bergen-Hohne unterstützte mich großzügig im Rahmen der ihr gegebenen Möglichkeiten. Ihr sei dafür nochmals gedankt.

\section{Bisherige Untersuchungen}

Die stratigraphische und regionale Einordnung der Endmoränen um den Falken-Berg war bisher nie schlüssig bewiesen, da spezielle, auf die Endmoränen ausgerichtete Untersuchungen bisher nicht vorlagen.

Stoller (1918), der Erstbearbeiter, beschrieb die Höhen um den Falken-Berg als „... geschlossenes Massiv aus der Ebene aufsteigender Endmoränen..." und ordnete sie seiner vorletzten Vereisung zu. WOLDSTEDT (1938) führte den Ausdruck „FalkenbergEnmoräne" ein und sah darin die Hauptfortsetzung des Rehburger Stadiums nördlich der Aller, aufgestaucht von einem aus dem Nordseegebiet kommenden, von NW nach SE vorrückenden Gletscher (S. 488). Ein Jahr später (Woldstedt 1939) folgte eine ausführliche Beschreibung: Ein saalezeitlicher Nordseegletscher, der durch norwegisches Eis nach $\mathrm{S}$ abgedrängt wurde, habe die Moränen aufgetaucht; parallel und in ihrer Fortsetzung verliefe im Hinterland in ca. $15 \mathrm{~km}$ Abstand (WoldSTEDT 1939: 238) eine kleinere Endmoräne desselben Nordseegletschers, die von Visselhövede bis Neuenkirchen zu verfolgen sei (Neuenkirchener Endmöräne).In einer späteren Arbeit stellte WoLDSTEDT (1950) die Falkenberg-Endmoräne in seine „Soltauer Staffel“, eine jüngere Staffel des Drenthe-Stadiums und ordnete sie einem von mehreren NE-streichenden Ästen seines Rehburger Stadiums zu. In seiner Tabelle 3 (S. 103) erschien das „Soltauer Stadium“ (hier nicht als Staffel bezeichnet) mit der Falkenberg-Endmoräne als jüngste von 3 Phasen des Drenthe-Stadiums. Niedermayer (1950a, 1950b) wies in seinen Berichten über die Kartierung der Blätter Fallingbostel und Bergen auf die auffallend frischen Formen der Endmoränen hin und sah sie als Bildungen eines drenthestadialen Rückzugsstadiums an. An keiner Stelle hatte er Grundmoräne oder deren Reste auf den Endmoränen feststellen können.

LüTTIG (1964) sah in seiner „Falkenberg-Staffel“ eine ältere Staffel der „Northeimer Phase“, jünger als die zur gleichen Phase gehörende „Ostenholzer Staffel“. Er faßte sie also als eine frühdrenthestadiale Bildung auf. Dieser Vorstellung folgte in den Grundzügen auch DUPHORN (in WoLDSTEDT 1974) und beschrieb die Falkenberg-Endmoräne als eine früh-drenthestadiale, vom Eise überfahrene Endmoräne, sah aber keine Möglichkeit, sie mit der Rehburger Stauchendmoräne in Verbindung zu bringen. 


\section{Form und Aufbau der Endmoränen}

Entgegen den Vorstellungen von WoLDSTEDT (1939) zeichnen sich drei nach verschiedenen Seiten geöffnete Endmoränenbögen ab, der Örbker, der Becklinger und der Kl. Bokkeler Endmoränenbogen.

1. Der OOrbker Endmoränenbogen umschließt die weitere Umgebung des heute zu Fallingbostel gehörenden Dorfes Örbke. Er führt vom Goldbocken-Berg im N

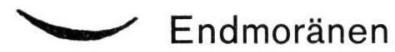

- - - Eisrandlage ohne Endmoränen

$\because \because$ Schmelzwasser-Ablagerungen Schüttungsrichtung

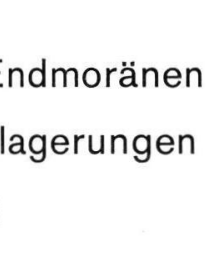

Fallingbostel

Örbke
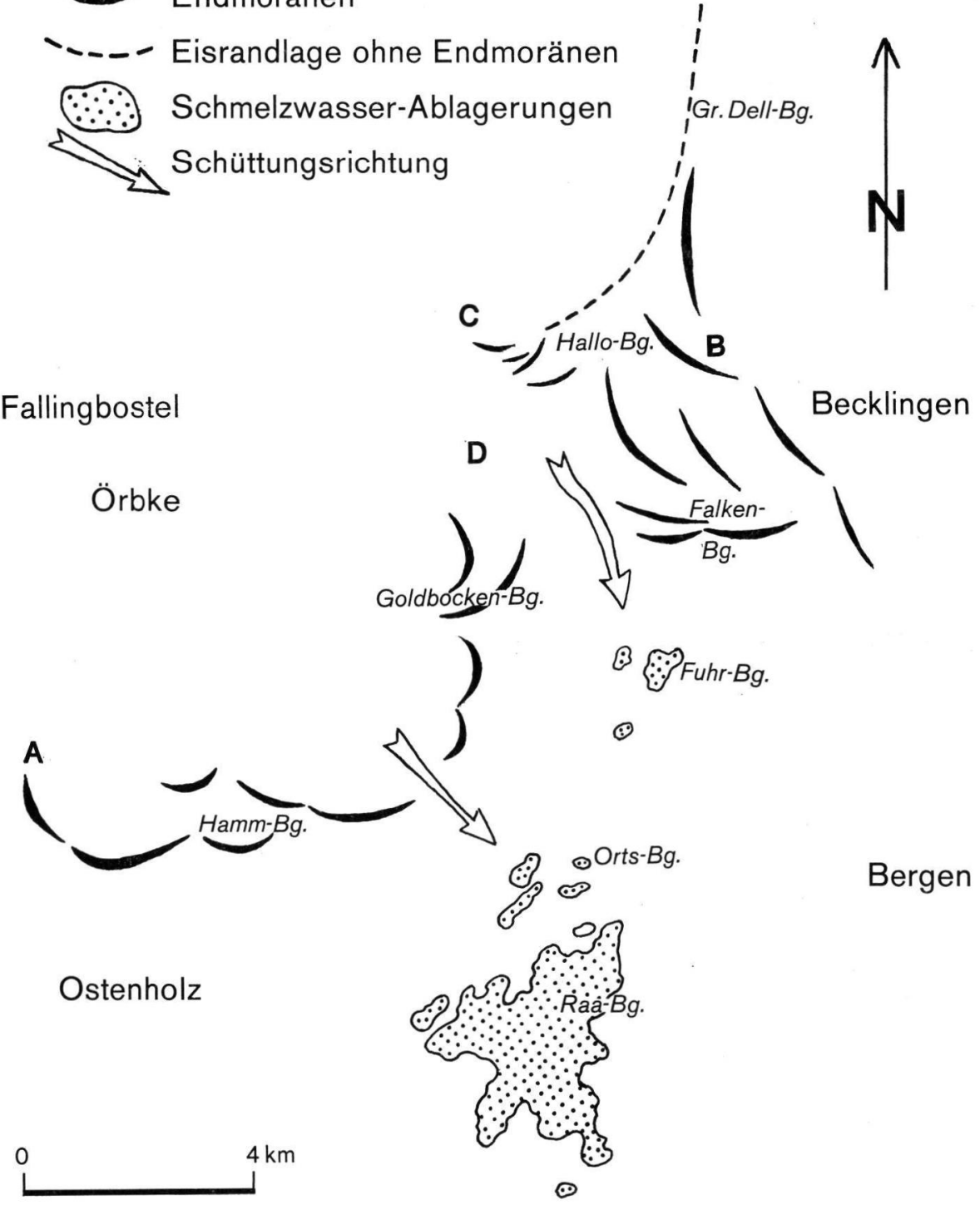

Abb. 1: Endmoränen, Eisrandlagen und Schmelzwasserablagerungen zwischen Bergen und Fallingbostel. - A Örbker Endmoränenbogen, B Becklinger Endmoränenbogen, C Kl. Bockeler Endmoränenbogen, D Achterberg-Senke. 
$(129,1 \mathrm{~m}$ üb. NN) nach S über den Tuten-Berg (115,8 $\mathrm{m}$ üb. NN) und den Ferschlan-Berg (88 $\mathrm{m}$ üb. NN), biegt dann nach W ab und führt über den Hamm-Berg (ca. $105 \mathrm{~m}$ üb. NN) bis nahe an die Autobahn Hannover-Hamburg (etwa $5 \mathrm{~km} \mathrm{~S}$ Fallingbostel) heran. Dort, wo dieser Endmoränenbogen nach W abknickt, besitzt er eine tiefe Kerbe, durch die heute der Hohebach fließt. Sein Tal ist in der Hauptstreichlinie des Endmoränenbogens auf etwa 60-62 m üb. NN eingetieft (s. Abb. 1).

Der Ớrker Endmorän en bogen besteht aus maximal 2 hintereinander gestaffelten Rücken. Die einzelnen Rücken zeigen einen steilen Abfall nach Innen (von W über NW nach N) und einen wesentlich flacheren nach Außen hin. Sie sind ganz überwiegend aus sehr feinen bis mittelkörnigen Sanden aufgebaut. Auch auf den Höhen selbst tragen sie meist nur eine wechselnd dichte Bestreuung mit Geröllen. Kompakte Grobsandund Kieslagen fehlen. Einzelne große Geschiebe treten lediglich in seinem nördlichen Teil, etwa vom Tuten-Berg nach $\mathrm{N}$, auf.

Die Höhen des Örbker Endmoränenbogens erheben sich maximal etwa $45 \mathrm{~m}$ über das halbkreisförmig umschlossene Becken von Örbke.

In diesem gesamten Endmoränenbogen gibt es kaum Aufschlüsse. Auch die wenigen, meist flachen, die vorhanden sind, zeigen keinerlei Hinweise auf irgendwelche Stauchungen.

An seinem N-Ende stößt der Orbker Endmoränenbogen an die Achterberg-Senke (s. unten). Seine Fortsetzung nach W ist unklar. Woldstedt (1939) gab keine an, LütTiG (1964) faßte ihn als zur Ostenholzer Staffel gehörig auf, die auf Bl. 3129 Walsrode nach NNW abbiegt und unter seine Walsröder Staffel abtaucht.

Die Achterberg-Senke, so benannt nach dem früher dort gelegenen Dorf Achterberg, trennt den Orbker Endmoränenbogen von den beiden nördlichen bzw. nordöstlichen Endmoränenbögen (s. Abb.1). Sie streicht NW-SE und ist an ihrer höchsten Stelle bis auf etwa $90 \mathrm{~m}$ üb. NN eingetieft. Sie wird vom Fischenbach durchflossen, der im unteren Teil dieses Tales zu einer Reihe von Fischteichen aufgestaut ist.

2. Nordöstlich der Achterberg-Senke treten in der näheren Umgebung des FalkenBerges bis zu 3 oder 4 hintereinander gestaffelte und mehrfach unterbrochene Höhenrükken auf, die sich in einen von NNW auf nahezu E umschwenkenden und nach NE geöffneten Endmoränenbogen einordnen lassen (Abb. 1). Da der Großteil von ihnen im „Becklinger Holz" liegt, wollen wir diesen Bogen als den Becklinger Endmor ä n e n b o g e n bezeichnen. Die einzelnen Rücken im Becklinger Endmoränenbogen erreichen Höhen bis zu $150 \mathrm{~m}$ üb. NN (Falken-Berg 146,9 m üb. NN, Haken-Berg 143,3 m üb. NN, Buller-Berg 139,6 $\mathrm{m}$ üb. NN). Er ist in sich wesentlich stärker gegliedert als der Örbker Fndmoränenbogen und wird bis zu $20 \mathrm{~m}$ höher als dieser.

Die Höhenrücken des B ecklinger Endmoränenbogens, mehrfach hintereinander gestaffelt und in sich stark gegliedert, zeigen auch wieder den steilen Abfall gegen die anzunehmenden Bewegungsrichtung des Eises, also nach NE, und einen flacheren nach SW. Die Rücken selbst sind wieder vorwiegend aus sehr feinen bis mittelkörnigen Sanden aufgebaut. Obenauf tragen sie gelegentlich kiesige Schichten, die, wie es z. B. ein Wasserriß am N-Hang des Falken-Berges zeigt, nur eine geringe Mächtigkeit besitzen. Kompakte Geröllagen und große Geschiebe fehlen. Stauchungen bzw. Hinweise darauf wurden nicht beobachtet. Jedoch gibt es nur sehr wenige und kaum flächenhafte Aufschlüsse.

Die Fortsetzung des Becklinger Endmoränenbogens nach $\mathrm{N}$ ist problematisch. Morphologisch gut erkennbare, als Endmoränen aufzufassende Rücken lassen sich nach $\mathrm{N}$ bestenfalls bis in die Gegend der Höhe 108,0, wenig südlich des Gr. Dellberges, verfolgen. Daran schließt sich nach $\mathrm{N}$ ein pultartiges, nach $\mathrm{E}$ abfallendes und von $\mathrm{W}$ und $\mathrm{E}$ her zertaltes 
Hochgebiet an. Woldstedt (1939) bezog dieses Gebiet mit in seine Falkenberg-Endmoräne ein und stellte in diesem Bereich in seiner Abb. 1 kurze, meist SW-NE-streichende, nach W bzw. NW geöffnete, leicht gebogene Endmoränen dar. Auf Blatt CC 3118 HamburgWest der Geologischen Übersichtskarte 1:200 000 verläuft vom Falken-Berg aus nach N durch dieses Gebiet eine Endmoräne, die ihre Fortsetzung in N-S-streichenden Rücken im Gebiet östlich Soltau findet.

Besondere Beachtung fanden bei diesen Deutungen die N-S-streichenden Rücken bei Lührsbockel mit ihren Kiesgruben (an der von Bergen nach Soltau führenden Bundesstraße 3, etwa $6 \mathrm{~km}$ SSE Soltau, bereits nördlich des auf Abb. 1 dargestellten Ausschnittes). Schuchт (1935, 1937), der diese Gruben im Rahmen seiner Kartierung des Blattes Soltau aufgenommen hatte, faßte die dort anstehenden Sande, Kiese und Gerölle als im Zuge von Endmoränen abgelagert auf, hatte jedoch Bedenken, die nach $S$ anschließende, bis zu $4 \mathrm{~km}$ breite und aus feinen Ablagerungen aufgebaute Hochfläche gleichfalls als Endmoräne aufzufassen. Niedermayer (1949) gab aus den Kiesgruben von Lührsbockel vorwiegend Grobkiese, im Hochgebiet südlich davon wesentlich freineres Material an.

Diese Befunde begründen mit die Annahme, daß die grobkiesigen Ablagerungen in den Rücken bei Lührsbockel nach Alter und Genese nicht unbedingt als die nördliche Fortsetzung unseres Becklinger Endmoränenbogens aufzufassen sind. Dieses ganze Gebiet um den Aschberg, den Dellberg und nördlich davon macht nach Morphologie und seinen Sedimenten weit eher den Eindruck eines Hochsanders nach GRIPP (1975), der hier an Stelle von Endmoränenbögen den Verlauf der Eisrandlage nach $\mathrm{N}$ markiert und der jünger ist als die Rücken bei Lührsbockel mit ihren Kiesen.

Woldstedt (1939) deutete alle Rücken im Falkenberg-Gebiet als Endmoränen, die zu einem aus NW kommenden Nordsee-Gletscher gehören. Als östlichen Gegenflügel faßte er den Mühlenberg-Zug, einen flachen Höhenrücken zwischen Wietzendorf und Bergen, auf (so benannt nach dem Mühlenberg bei Widdernhaus).

Nach Beobachtungen in einer heute weitgehend verfüllten Sandgrube auf dem Sitternberg bei Nindorf und in einer Sandgrube etwa $1 \mathrm{~km}$ SE Bleckmar an der Eisenbahnlinie Bergen-Soltau zeigen die kreuzgeschichteten Sande des Mühlenberg-Zuges vorwiegend ein Einfallen nach S, also in Richtung seiner Längserstreckung. Es dürfte sich also hier nicht um eine Endmoräne, sondern eher um ein Os handeln, wie auch schon bei LǘtTIG (1964) dargestellt.

Wir vermuten die Fortsetzung des Becklinger Endmoränenbogens nach SE bzw. nach $\mathrm{E}$ in den NW-SE-streichenden Höhen bei Bollersen auf Bl. 3225 Offen (Goldberg und Höhe 83,3), die sich im Schwarzen Berg bei Sülze und weiter östlich im Rübenberg und im Zitronenberg bei Rebberlah fortsetzen (vgl. auch Woldstedt 1939; LütTig 1964). Wenn auch zwischen den südöstlichen Ausläufern des Becklinger Endmoränenbogens bei Bleckmar und dem Goldberg bei Bollersen eine nicht belegte Spanne von etwas mehr als $4 \mathrm{~km}$ liegt, so scheint dieser Konnektionsversuch (Abb. 3) vom Gesamtkonzept her diskussionswürdig.

3. Im Gebiet des ehemaligen Dorfes Kl. Bockel, etwa $4 \mathrm{~km}$ SE Dorfmark, deuten einige flache und kurze Rücken einen weiteren Endmoränenbogen an, den K1. B o ck e le r Endmor än enbogen. Mit maximalen Höhen von 100 bis $110 \mathrm{~m}$ üb. NN wirken sie im Vergleich zu den bisher beschriebenen ausgesprochenen dürftig und weniger überzeugend. Ihre Längsstreckung beträgt nur etwa $1 \mathrm{~km}$. Dieser Kl. Bockeler Endmoränenbogen besteht aus wenigen kurzen, hintereinander gestaffelten, meist SW-NE-streichenden Rücken, die eine leicht bogige Form zeigen und deren NW-Hang wesentlich steiler als der Abfall nach SE ist. In der petrographischen Ausbildung scheint er weitgehend den bisher beschriebenen Endmoränenbögen zu entsprechen. Allerdings ist dieser Teil des 
Blattes Fallingbostel auf der Kartierung von Niedermayer (1950a) ausgespart. Die einzelnen Rücken lassen sich von der Achterberg-Senke etwa $1 \mathrm{~km}$ weit nach NE verfolgen und laufen dann aus.

Der Kl. Bockeler Endmoränenbogen dürfte einen Eislobus andeuten, der von NW heranrückte (s. WOLDSTEDT 1939) und dessen Stirn von SW nach nahezu N bogig verlief. Der Außenrand dieses Lobus wird nördlich der kurzen Rücken durch den Steilanstieg des Falkenberg-Hochgebietes gegenüber der unmittelbar nordwestlich vorgelagerten und teilweise vermoorten Senke markiert. Zu keinem der beschriebenen Endmoränenbögen dürfte der NW-SE-streichende Rücken gehören, der mit dem Möhl-Berg nahe der ehemaligen Dorfstelle Achterberg beginnt und unsere Achterberg-Senke zeitweilig nach SW begrenzt (s. Abb.1). Er wird von Grundmoräne überdeckt, muß also älter als die hier bisher beschriebenen Endmoränenbögen sein.

In keinem der z. T. sehr schön ausgebildeten Endmoränenbögen sind bisher Stauchungen beobachtet worden. Alle diese Rücken bestehen aus relativ feinkörnigem Material. Lediglich obenauf sind sie mehr oder weniger dicht mit Geröllen oder kleinen Geschieben bestreut. Kompakte Kieslagen auf den Kämmen, vielleicht 2-3 m mächtig, waren nur auf einzelnen Rücken des Becklinger Endmoränenbogens zu beobachten. Vermutlich liegen in diesem ganzen Bereich nur Satz-Endmoränen in relativ feinkörniger Abbildung vor (Feinkorn-Satz - Endmoränen, GRIPP 1975). Auch eine Deutung als „Hochsander“ (GRIPP 1975) dürfte für Teile von ihnen zutreffen.

\section{Das Gebiet hinter den Endmoränenbögen}

Der Ớrker Endmoränenbogen umgrenzt ein Becken, in dem die Ortschaft Órbke liegt und das dadurch gekennzeichnet ist, daß an der Oberfläche bzw. in nur geringer Tiefe zwei Geschiebelehme bzw. Geschiebemergel auftreten, die durch mehrere Meter mächtige glazifluviatile Sande getrennt sind und die beide vermutlich dem DrentheStadium zugehören.

Nach der Kartierung von Niedermayer (1950a) treten am südlichen und östlichen Rand des Beckens glazifluviatile Sande in einem meist schmalen Streifen zu Tage. Beckenwärts legt sich darüber eine Grundmoräne, die nach dem Kartenbild bis zu $5 \mathrm{~m}$ mächtig $\mathrm{zu}$ werden scheint.

Nach Bohrungen besonders in der näheren Umgebung von Ơrbke folgt unter dieser obersten Grundmoräne und unter glazifluviatilen Sanden in wenigen Metern Tiefe oft eine weitere Grundmoräne (z. B. Bohrungen Hy $44=$ Órbke 9 und Hy $49=$ Órbe 10, Bl. Fallingbostel; Archiv Nieders. Landesamt $\mathrm{f}$. Bodenforschung). Nach Ausbildung und Lagerungszusammenhang setzen wir die untere der beiden Grundmoränen der Grundmoräne gleich, die im weiteren Vorland der Endmoränenbögen auftritt und in der wir die Drenthe-Hauptmoräne sehen (s. Abb. 2). In größerer Tiefe (20 bis $30 \mathrm{~m})$ folgt darunter oft eine weitere, vermutlich elsterzeitliche Grundmoräne (vgl. LANG 1980: Erläuterungen zu Bl. 3224 Westenholz).

In diesem Zusammenhang ist ein Befund aus der Sandgrube auf dem Marquardsfeld unmittelbar am Ortsrande von Órbke interessant, wie man ihn vor etwa 10 Jahren beobachten konnte.

Die Sandgrube ist in einem NW-SE-streichenden Rücken angelegt. In der damals 8-10 $\mathrm{m}$ tiefen Grube konnte man unter einer dünnen Grundmoränendecke gestauchelte Sande und Kiese beobachten, in die auch älterer Geschiebelehm mit eingeschaltet war. Der eingestauchte Geschiebelehm war graubraun, relativ hell und enthielt reichlich Feuerstein. 


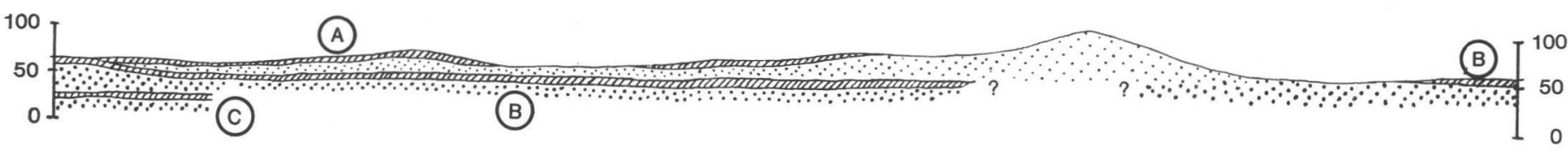

WIIA Geschiebelehm oder Geschiebemergel

\section{(A) jüngere Drenthe-Moräne (B) Drenthe-Hauptmoräne}

(C) Elster-Moräne (?)

$\therefore$ Glazifluviatiler Sand,jung-drenthezeitlich

$\because \because$ Glazifluviatiler Sand,haupt-drenthezeitlich und älter

Abb. 2: Stark schematisiertes WNW-ESE-Profil durch den Ơrbker Endmoränenbogen und sein 
Er entsprach in seinem Aussehen nicht dem vermutlich elsterzeitlichen Geschiebelehm bzw. Geschiebemergel der südlich anschließenden Blätter 3224 Westenholz (LANG 1980) und 3225 Offen (LANG 1983) der Geol. Karte von Niedersachsen $1: 25000$, sondern weitmehr der drenthestadialen Hauptmoräne, die auf den südlich anschließenden Blättern, auch südlich der Aller-Niederung, weit verbreitet ist.

Ein völlig anderes Bild zeigt das nördliche und nordöstliche Rückland des B e ck linger Endmoränenbogens, beiderseits der alten Straße von Bergen über Becklingen nach Soltau. Es liegt in Höhen zwischen 80 und etwas mehr als $100 \mathrm{~m}$ üb. NN und ist von $\mathrm{W}$ und $\mathrm{E}$ her leicht zertalt. Trotz dieser Zertalung erkennt man noch die pultartige, nach E bzw. NE leicht geneigte Hochfläche. Flache Aufschlüsse, Wegeböschungen und eine Reihe von Handbohrungen haben unter einer meist dünnen Geröll- oder Geschiebebestreuung nur feinst- bis mittelkörnige, recht eintönige Sande gezeigt. Grundmoräne oder große Geschiebe fehlen.

Für das unmittelbare Rückland des K1. B ockeler Endmoränenbogens liegen nur wenige geologische Daten vor. Es ist nahezu beckenartig eingesenkt, teilweise vermoort und enthält eine Reihe von Teichen. An der Oberfläche stehen weit verbreitet sandige weichselzeitliche Schichten an. Etwa westlich der Eisenbahnlinie DorfmarkSoltau setzt dann wieder Grundmoräne in flächenhafter Verbreitung ein (NIEDERMAYER 1949, 1950a).

\section{Das Gebiet vor den Endmoränen und seine Schmelzwasserbildungen}

Im weiteren Vorland der Endmoränenbögen auf den südlich anschließenden Blättern 3224 Westenholz (LANG 1980) und 3225 Offen (LANG 1983) der Geol. Karte von Niedersachsen 1:25000 tritt nur eine drenthe-stadiale Grundmoräne auf, die vielfach bis auf Reste erodiert ist. Eine weitere, insbesondere in der Aller-Niederung oder auf Blatt Offen unmittelbar über anstehendem Tertiär oder in Verbindung mit subglaziären Rinnen auftretende tiefere Grundmoräne ist nach ihrem gesamten Habitus und nach den Lagerungsverhältnissen der Elster-Kaltzeit zuzuordnen.

Von besonderem Interesse sind die Reste eines großen kiesig-grobsandigen Sedimentfächers, der sich vom Fuhr-Berg und Kuhlen-Berg, also unweit der Endmoränenbögen, über etwas mehr als $8 \mathrm{~km}$ nach $\mathrm{S}$ bis nahe heran an die Aller-Niederung verfolgen läßt. Wir sehen darin die Ablagerungen von Schmelzwässern aus den Gletscherloben unmittelbar hinter unseren Endmoränenbögen. Im NE-Teil des Blattes 3224 Westenholz der GK 25 (LANG 1980) und im westlichen Grenzgebiet des östlichen Nachbarblattes 3225 Offen (LANG 1983) treten sie auf einer Fläche von etwa $3 \times 2 \mathrm{~km}$ auf. Diese Kiese und Grobsande erreichen hier eine Mächtigkeit bis etwa $5 \mathrm{~m}$; ihre Unterkante steigt von ca. $50 \mathrm{~m}$ üb. NN am südlichen Ende bis auf etwa $65 \mathrm{~m}$ üb. NN am nördlichen Blattrand an, also etwa um $15 \mathrm{~m}$ auf $3 \mathrm{~km}$. Die Kartierungen und eine Bohrung haben gezeigt, daß der als „Oberer Schmelzwassersand“ angesprochene Sedimentfächer (LANG 1980) von der einzigen dort auftretenden drenthestadialen Grundmoräne (Drenthe-Hauptmoräne) unterlagert wird. Auf dem Fächer selbst ließen sich keine Anzeichen für eine Grundmoräne oder auch Stauchung durch Überfahrung finden.

Weiter nach $\mathrm{N} \mathrm{zu}$ ist dieser Fächer großenteils erodiert. Hier sind nur noch einzelne Kuppen mit grobsandig-kiesigem Material vorhanden, in einer Mächtigkeit von mehreren Metern. Es wird in der Regel von einem sehr erosionsanfälligen feinsandigen und schluffigen Material unterlagert.

Über den Orts-Berg (Unterkante der kiesigen Schichten etwa bei 70-75 m üb. NN), Sechsstücker-Berg (Unterkante bei etwa $82-85 \mathrm{~m}$ üb. NN) und Kuhlen-Berg und Fuhr- 
Berg (Unterkante bei etwa 92-95 m üb. NN) läßt sich dieser Schüttungsfächer bis nahe an die Endmoränenbögen heran verfolgen.

Die Entfernung vom Fuhr-Berg bis zum distalen Ende unseres Grobsand- und Kiesfächers beträgt etwas mehr als $8 \mathrm{~km}$; seine Unterkante steigt über diese Distanz um ca. $42-45 \mathrm{~m}$ an, also um $5 \mathrm{~m}$ oder etwas mehr auf $1 \mathrm{~km}$ (vgl. S. 38).

Die starke Erosion dieser Schmelzwasserablagerungen dürfte durch die Natur der unterlagernden Schichten bedingt sein. Diese bestehen zu einem großen Teil aus vielfach schluffigen Feinsanden, die der Erosion nur einen geringen Widerstand entgegensetzen. Auch heute kann man immer wieder beobachten, welch tiefe Runsen in diesem Gebiet, sobald die Vegetation beschädigt ist, auch nach kurzen Starkregen entstehen und welche Schwemmfächer dann hangabwärts bzw. in Senken hinein geschüttet werden. Die hangenden Schichten fallen dann der Abtragung mit anheim.

In einer 1981 erschienenen Arbeit hat ScHunke interessante Daten zur Erosionsleistung von Flüssen in Zentral-Island gegeben, wo heute klimatische Verhältnisse vergleichbar denen während des Warthe-Stadiums und der Weichsel-Kaltzeit in NW-Deutschland herrschen. Im Flußgebiet des Blanda, über das Schunke (1981) berichtet, stehen über tertiären und quartären Eruptiva auf einem Plateau in $500-700 \mathrm{~m}$ üb. NN glaziale und glazifluviale Sedimente an, die stellenweise von einem lößartigen Material überdeckt werden. Sie tragen weitflächig keine Vegetationsdecke. Für dieses Gebiet wurde eine flächenhafte Erosion von etwa $700 \mathrm{~mm}$ in 1000 Jahren ermittelt.

In der näheren Umgebung des Fuhr-Berges reicht heute die Abtragung bis etwa $30 \mathrm{~m}$ unter die Unterkante der Schmelzwasserablagerungen herab. Nahmen wir auch hier eine Erosionsleistung von $700 \mathrm{~mm}$ in 1000 Jahren an, was angesichts der hier anstehenden, sehr erosionsempfindlichen Fein- und Feinstsande ein noch relativ niedriger Wert sein dürfte, so genügte eine Zeit von größenordnungsmäßig 45000 Jahren, um $30 \mathrm{~m}$ dieses Sediments flächenhaft abzutragen. Diese Zeit stände während des Warthe-Stadiums und während der Weichsel-Kaltzeit durchaus zur Verfügung.

Zwei Schotteranalysen vom Fuhr-Berg (nördlicher Teil des SchmelzwassersedimentFächers) und vom Raa-Berg (südlicher Teil des Fächers) zeigen in ihrer Zusammensetzung eine sehr gute Übereinstimmung (s. Tab. 1).

Tab. 1: Schotterproben vom Raa-Berg und vom Fuhr-Berg. Fraktion 6,3 bis 12,5 mm

\begin{tabular}{|c|c|c|c|c|}
\hline & $\begin{array}{r}\text { Raa-Berg } \\
\text { R } 3556780 \text {, }\end{array}$ & $\begin{array}{l}2 \text { m Tiefe } \\
\text { H } 5849510\end{array}$ & $\begin{array}{r}\text { Fuhr-Berg, } \\
\text { R } 3557960,\end{array}$ & $\begin{array}{l}\text {, 0,2 m Tiefe } \\
\text { H } 5855150\end{array}$ \\
\hline Nordisch (Summe) & 248 & $95,5 \%$ & 312 & $96,3 \%$ \\
\hline Kristallin & 95 & $36,2 \%$ & 161 & $49,7 \%$ \\
\hline Sandstein u. Quarzit & 72 & $27,5 \%$ & 55 & $17 \%$ \\
\hline Ton- u. Schluffstein & 2 & $1 \%$ & - & - - \\
\hline Feuerstein & 79 & $30,5 \%$ & 96 & $29,6 \%$ \\
\hline Einheim.-mesozoisch & - & -- & - & --- \\
\hline Einheim.-paläozoisch & - & - - & - & --- \\
\hline Quarz & 10 & $3,5 \%$ & 12 & $3,7 \%$ \\
\hline Unbest. & 4 & $1 \%$ & - & --- \\
\hline Gesamt & 262 & $100 \%$ & 324 & $100 \%$ \\
\hline
\end{tabular}


Beide Proben sind durch einen sehr hohen Anteil an nordischem Kristallin und an Feuerstein und das Fehlen einheimisch-südlichen Materials gekennzeichnet. Die Zusammensetzung stützt die Vorstellung eines von Schmelzwassern geschütteten Sedimentfächers und auch die Auffassung, daß hier ein einheitlicher, vielleicht von 2 Schmelzwasserflüssen genährte Schüttungskörper vorliegt.

Wie die Abb. 1 zeigt, bietet sich als Ausgangspunkt dieses Kiessandfächers die Achterberg-Senke an, der Zwickel zwischen dem Ớrbker und dem Becklinger Endmoränenbogen. Dort, wo die beiden Endmoränenbögen am nächsten zusammenkommen, beträgt die heutige Geländehöhe knapp $90 \mathrm{~m}$ üb. NN. Unter der Annahme, daß auch hier nachdrenthestadial kräftig erodiert worden ist, kommen wir leicht auf eine Ausgangshöhe in diesem Ursprungsgebiet unseres Schüttungsfächers von etwa $100 \mathrm{~m}$, wie er nach dem beobachteten Gefälle und der Unterkante der Sande und Kiese auf dem Fuhr-Berg zu fordern ist.

Niedermayer (1950a) sah im Einschnitt des heutigen Hohebach-Tales durch den hier nach W abknickenden Örbker Endmoränenbogen unmittelbar nördlich der „Sieben Steinhäuser" ein altes Gletschertor. Das diesem Durchbruch nächstgelegene Vorkommen von Schmelzwasserablagerungen, etwa $2 \mathrm{~km}$ von hier in SW-Richtung entfernt, zeigt die Unterkante der Kiessande in etwa $68 \mathrm{~m}$ üb. NN, etwa 6-8 $\mathrm{m}$ höher als die heutige Talsohle des Hohebach-Tales im Durchbruch. Es fällt nicht schwer anzunehmen, daß der Hohebach sich in post-drenthestadialer Zeit so weit eingetieft hat (also etwa um 15-18 m), daß sich auch in der Höhe ein Zusammenhang ergibt, der die Annahmen von Niedermayer (1950a) bestätigt. Auch dieser Betrag liegt in der Größenordnung, die man nach den Untersuchungen von Schunke (1981) in Island für eine linienhafte Erosion erwarten kann. Demnach könnte dieser Fächer auch aus 2 Quellen gespeist worden sein (vgl. Abb. 1).

Von Interesse ist in diesem Zusammenhang ferner die Häufung großer Geschiebe in der Achterberg-Senke bzw. unweit ihres südlichen Ausganges und deren rasche Abnahme weiter nach S bzw. SW. Man könnte sie als einen Hinweis auf die unmittelbare Nähe des Eisrandes deuten.

Wie bereits eingangs dieses Abschnittes betont, haben wir hier also einen sich etwa $8 \mathrm{~km}$ weit nach S hinziehenden, von Schmelzwassern abgelagerten Fächer aus Kiesen und Sanden vor uns, der an die Endmoränenbögen ursächlich gebunden ist. Die Hauptschüttung dürfte aus der Achterberg-Senke heraus erfolgt sein, wo die Endmoränenbögen nahezu zusammenstoßen. Es ist auch daran zu denken, daß ein Teil der Schüttung aus einem Gletschertor im heutigen Tal des Hohebaches unmittelbar nördlich der „Sieben Steinhäuser"kam.

\section{Das Alter der Endmoränen}

Alle bisherigen Bearbeiter dieses Gebietes haben die Frische der Oberflächenformen betont, wie sie im Grunde für das Altmoränengebiet untypisch sind. Sie sprechen mit gegen eine Überfahrung durch das nordische Inlandeis, die wahrscheinlich zu einer teilweisen Einebnung geführt hätte.

Bei den von Niedermayer (1950a, b) durchgeführten Übersichtskartierungen und bei gezielten eigenen Untersuchungen haben sich keinerlei Hinweise auf Grundmoräne bzw. deren Überreste auf den Endmoränenbögen finden lassen. Hingegen ist sehr wahrscheinlich, daß hinter dem Órbker Endmoränenbogen in der weiteren Umgebung von OÓrbke 2 drenthestadiale Grundmoränen auftreten (vgl. Abschnitt über das Rückland des OOrbker Endmoränenbogens) und daß im Vorland im Gebiet der Blätter 3224 Westenholz (LANG 1980) und 3225 Offen (LANG 1983) der Geol. Karte 1:25000 nur eine drenthestadiale Grundmoräne vorhanden ist (Abb. 2). 
Wie bei Duphorn (in Woldstedt 1974: 224) nochmals zusammengestellt, zeigt das Drenthe-Stadium in der Lüneburger Heide folgende Gliederung:

Jüngerer Geschiebemergel,

Schmelzwassersand,

Alterer Geschiebemergel (Drenthe-Hauptmoräne).

Diese Gliederung trifft (mit Einschränkungen) auch für unseren Raum zu. Der ältere Geschiebemergel läßt sich weit nach S verfolgen. Der Schmelzwassersand wird durch den nach S geschütteten Sand- und Kiesfächer repräsentiert, der jüngere Geschiebemergel ist in dem vom Ớrbker Endmoränenbogen umschlossenen Gebiet vorhanden.

Wenngleich das Rückland des Becklinger Endmoränenbogens anders aussieht als z. B. das des Örbker Endmoränenbogens, so sprechen doch die Oberflächenformen, das Fehlen einer Grundmoräne auf all diesen Endmoränenrücken und die Haupt-Schmelzwasserschüttung aus der Achterberg-Senke, dem Zwickel zwischen den Endmoränenbögen, für eine gleichzeitige Entstehung der drei Endmoränenbögen.

Wir nähern uns damit wieder der von Woldstedt $(1938,1939)$ und von NiederMAYER (1950b) gegebenen zeitlichen Einstufung, die in der Falkenberg-Endmoräne eine spätdrenthezeitliche Bildung bzw. die eines drenthestadialen Rückzugsstadiums sahen.

\section{Regionale Einordnung und Versuch einer Deutung}

Woldstedt $(1938,1939,1950)$ sah in seiner Falkenberg-Endmoräne eine Hauptfortsetzung der Rehburger Stauchendmoräne nördlich der Aller bzw. einen von mehreren NE-streichenden Ästen des eben erwähnten Endmoränenzuges. Nach LüTTIG (1964) zieht die Falkenberg-Staffel nach ENE und taucht unter die Warthe-Endmoräne. Die Fortsetzung nach NW ist unklar.

Da die Rehburger Stauchendmoräne vom nördlichen Inlandeis überfahren worden ist (MEYER 1980), scheidet allein schon daher eine Verbindung unserer Endmoränenbögen mit der Rehburger Stauchendmoräne aus. Auch aus der regionalen Anordnung wäre diese Verbindung nur schwer zu verstehen (s. Abb. 3). Gewisse Parallelen in der Form und im Aufbau zeigen sich mit dem Gebiet um den Brelinger Berg nahe Mellendorf (LANG 1964); eine unmittelbare Verbindung läßt sich jedoch auch hier (s. Abb. 3) kaum vertreten.

Bereits ScHuснт (1936) hatte vermutet, „... daß sich in diesem diluvialen Hochgebiet präexistente Reliefformen widerspiegeln,..." (S. 2). Die Kiesrücken nahe Lührsbockel (vgl. S. 35) und mit Grundmoräne überzogene endmoränenartige Rücken nahe der ehemaligen Dorflage Achterberg (vgl. S. 36) könnten in diesem Sinne gedeutet werden. Was sich im Kern des Falkenberg-Hochgebietes selbst noch verbirgt, ist nirgends erschlossen.

Die folgende Möglichkeit einer Deutung der Endmoränenzüge im Untersuchungsgebiet zeichnet sich ab:

Eine Klimaverschlechterung gegen Ende des Drenthe-Stadiums reaktivierte das bereits abtauende Inlandeis und ließ es vorstoßen, in unserem Bereich in 3 Loben, in zweien aus NW und einem aus NE. Das heutige Falkenberg-Hochgebiet ragte damals bereits über seine Umgebung hinaus, vielleicht als Folge von Stauchungen während früherer Eisvorstöße. Der kräftige und den westlichen Loben wahrscheinlich voraneilende NE-Lobus besaß noch die Kraft, an bzw. auf diesem Widerlager seine Endmoränenrücken aufzubauen, die nach $\mathrm{N} \mathrm{zu}$ wahrscheinlich in Hochsander anstelle von Endmoränen übergehen.

Der etwas zögernd aus NW vorrückende und schwächere Kl. Bockeler Eislobus stieß im Gebiet nördlich des Hallo-Berges unmittelbar an den schon vorher vorgerückten Becklinger Eislobus, etwa entlang der heutigen Westgrenze des Falkenberg-Hochgebietes. Nur an seinem südlichen Ende hatte er Raum, um einige kleine Endmoränenrücken zu formen (s. Abb. 1). 

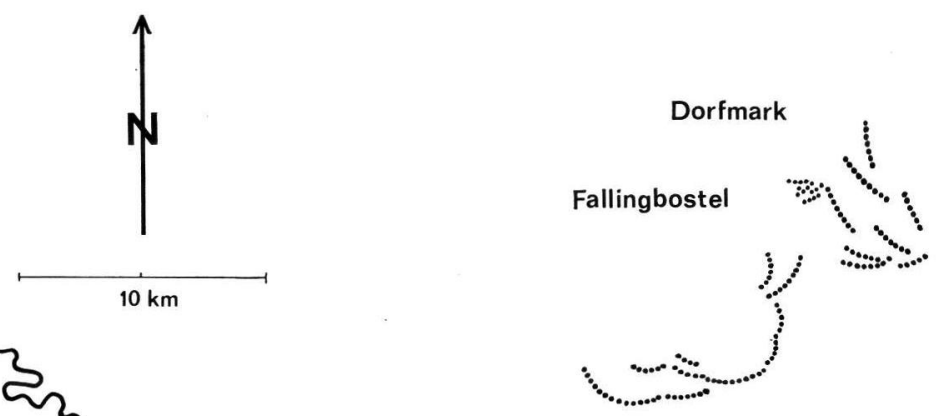

Bergen
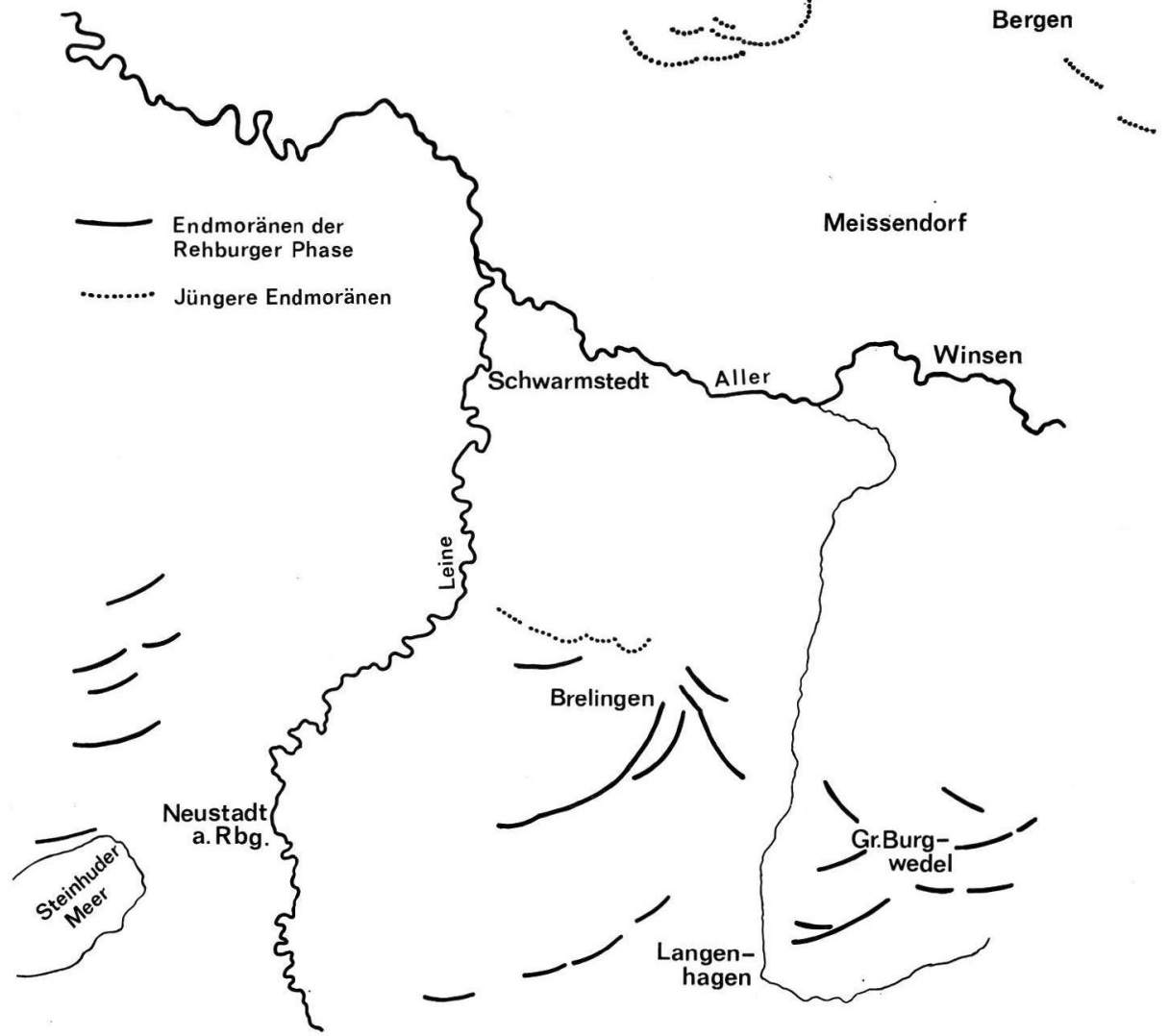

Abb. 3: Ơrbker, Becklinger und K1. Bockeler Endmoränenbogen und deren Lage zu Endmoränen im Gebiet nördlich von Hannover.

Der etwas weiter südlich gelegene Örbker Eislobus konnte sich frei und ungehindert entfalten und einen noch heute sehr gut erhaltenen Endmoränenbogen aufbauen.

Durch die Achterberg-Senke und möglicherweise eine Senke im Gebiet des heutigen Hohebaches flossen die Schmelzwässer dieser Eisloben nach S bzw. SE ab und lagerten ihre Sand- und Kiesfracht in einem Fächer ab, der heute in Resten erhalten ist, bis nahe an die Aller-Niederung heranreicht und dessen Unterkante ein Gefälle von etwa 5\%o zeigt. Mit der Möglichkeit, daß die Schmelzwässer im Gebiet der heutigen Aller-Niederung nach NW hin abflossen, ist zu rechnen. Weiter nach $S$ ist das Eis dann nicht mehr vorgestoßen. 


\section{Schriftenverzeichnis}

Gripp, K. (1975): Hochsander - Satzendmoräne - Endmoränenvertreter. - Z. Geomorph. N. F., 19 (4): 490-496, 1 Photo, 3 Abb.; Berlin, Stuttgart.

LANG, H. D. (1964): Uber glaziäre Stauchungen in den Mellendorfer und Brelinger Bergen nördlich von Hannover. - Eiszeitalter u. Gegenwart, 15: 207-220, 4 Abb., 1 Tab.; Öhringen/ Württ.

- (1980): Geol. Karte Niedersachsen, Erl. Bl. 3224 Westenholz: 98 S., 12 Abb., 5 Tab., 5 Kt.; Hannover.

- (1983): Geol. Karte Niedersachsen, Erl. Bl. 3225 Offen. — [Im Druck.]

Lütrig, G. (1966): Prinzipielles zur Quartärstratigraphie. — Geol. Jb., 82: 177-202, 1 Abb., 1 Taf.; Hannover. - [Sonderdrucke 1964.]

Míeyer, K.-D. (1980): Zur Geologie der Dammer und Fürstenauer Stauchendmoränen (Rehburger Phase des Drenthe-Stadiums). - Festschr. Gerhard Keller: 83-104, 3 Abb., 1 Tab., 1 Taf.; Osnabrück (Wenner).

Niedermayer, J. (1949): Erläuterungen zu Blatt Soltau 1:25000. - Man. Arch. Nieder. L.-Amt Bodenforsch.: 5 S.; Hannover. - [Unveröff.]

- (1950a): Erläuterungen für das Meßtischblatt Fallingbostel 1:25000: Arch. Nieder. L.-Amt Bodenforsch.: 8 S.; Hannover. - [Unveröff.]

- (1950b): Erläuterungen zu Blatt Bergen 1:25000. - Man. Arch. Nieders. L.-Amt Bodenforsch.: 9 S.; Hannover. - [Unveröff.]

Stoller, J. (1918): Geologischer Führer durch die Lüneburger Heide. - 168 S., 38 Fig., 8 Taf.; Braunschweig (Vieweg).

Woldstedt, P. (1938): Uber Vorstoß- und Rückzugsfronten des Inlandeises in Norddeutschland. - Geol. Rdsch., 29, 6: 481-490, 2 Abb.; Stuttgart.

- (1939): Über Endmoränen in der südlichen Lüneburger Heide. - Abh. Naturw. Ver. Bremen, 31: 236-246, 3 Abb.; Bremen.

- (1950): Norddeutschland und angrenzende Gebiete im Eiszeitalter: 464 S., 97 Abb., 12 Tab.; Stuttgart (Koehler).

- (1974): Norddeutschland und angrenzende Gebiete im Eiszeitalter. 3. Aufl., neu bearb. u. herausgegeb. von K. Duphorn: 500 S., 91 Abb., 26 Tab.; Stuttgart (Koehler).

Schuснт, F. (1935): 2. Bericht über die geologisch-agronomische Aufnahme des Blattes Soltau (Hannover) - Sommer 1935. - Ber. Arch. Nieders. L.-Amt Bodenforsch.; 8 S.; Hannover. [Unveröff.]

- (1937): Erläuterungen zu Blatt Soltau. - Man. Arch. Nieders. L.-Amt Bodenforsch.: 32 S., 9 Phot.; Hannover. - [Unveröff.]

Schunke, E. (1981): Abfluß und Sedimenttransport im periglazialen Milieu Zentral-Islands als Faktoren der Talformung. - Die Erde, 112: 197-215, 10 Fig., 1 Tab., 2 Bilder; Berlin.

Manuskript eingegangen am 10. 2. 1983. 
Hans Dietrich Lang 